ISSIN 2229-3531
JCIT Vol. 9(6), 70-74 (2018). Periodicity-2-Monthly

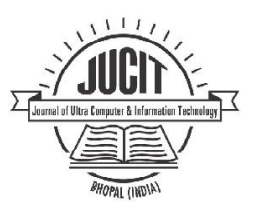

Estd. 2010
JOURNAL OF COMPUTER \& INFORMATION TECHNOLOGY

An International Open Free Access Peer Reviewed Research Journal of Computer Science Engineering \& Information Technology

website:- www.compitjournal.org

\title{
A CloudSim-based Analysing for Cloud Computing Environments and Applications
}

\author{
BHAVANI KANWAR ${ }^{1}$, DASHRATH SINGH ${ }^{2}$, SUMAN SINGH $^{3}$ and KRITI ARYA \\ ${ }^{1}$ M. tech (CSE), SIET, Sikar (Rajasthan), (India) \\ ${ }^{2}$ Lecturer (CSE), BTTI, Pilani (Rajasthan), (India) \\ ${ }^{3}$ Assistant Professor (CSE), SIET, Sikar (Rajasthan), India \\ ${ }^{4}$ M.tech (ECE), BRCM CET, Bahal (Haryana), (India)
}

Email address of Corresponding Author: ${ }^{1}$ bhavaninirwan@gmail.com, ${ }^{2}$ dashrath.btti@ gmail.com,

\author{
3 er.suman05@gmail.com, ${ }^{4}$ kritikarya16@gmail.com \\ http://dx.doi.org/10.22147/jucit/090601
}

Acceptance Date 26th December 2018

Online Publication Date 27th December, 2018

\begin{abstract}
Cloud Computing is the rising field of newest computing in this day and age. The cloud computing is based on the five essential characteristics and its three prime services (Software as a Service, Platform as a Service, Infrastructure as a Service). The major goal of cloud computing is to provide the resources as a services to the cloud consumers or clients. The new concept of Federated Cloud Computing in which multiple datacenters are distributed over different geographical regions. Since the evolution of Cloud Computing: Load balancing and management, energy efficient management, Virtual Machine(VM) migration, service brokerage policies, cost modeling, security issues and trust management are popular research topics in the field. The process of deployment of real cloud environment for testing or for commercial use is extremely costly. Cloud simulators help to model various cloud applications and it is very easy to analyze. In this survey, we used the CloudAnalyst, GUI Java based simulator with their overview are presented so it can be easily decided which one is suitable for particular research topic. And also the survey on the service broker policy, its issues and available solutions are presented. In this thesis we focused on performance evaluation of large data centers using some parameters like cost, service broker policies, User base, Number of physical machines in data centers, Data centers processing time, load balancing policies. Because there is always been the requirement to select appropriate datacenter so that further tasks for processing the request should be carried out with efficiency in least response time. So the issue of selecting appropriate datacenter which is known as service broker policy is kind of important. We also demonstrate the trust as factor majorly used between the cloud service provider and cloud consumers.

Key words : Cloud Computing, Cloud Simulators, Large Data Centers, Trust Management in Cloud, Service Broker Policies.
\end{abstract}

This is an open access article under the CC BY-NC-SA license (https://creativecommons.org/licenses/by-nc-sa/4.0) 


\section{Introduction}

Cloud computing is an area that is experiencing a rapid advancement both in academia and industry. This technology, which aims at offering distributed, virtualized, and elastic resources as utilities to end users, has the potential to support full realization of "computing as utility" in the near future ${ }^{9}$. Along with the advancements of the Cloud technology, new possibilities for Internetbased applications development are emerging. These new application models are based in two parties: in one side, there are the cloud service providers that are willing to provide large-scale computing infrastructure at a price based primarily on usage patterns. It eliminates the initial high-cost, for application developers, of environment set up an application deployment environment. On the other side there are large-scale software systems providers, which develop applications such as social networking sites and e-commerce, which are gaining popularity on the Internet. These applications can benefit greatly of Cloud infrastructure services to minimize costs and improve service quality to end users. Previously, development of such applications required acquisition of servers with a fixed capacity able to handle the expected application peak demand, installation of the whole software infrastructure of the platform supporting the application, and configuration of the application itself.

Furthermore, servers were underutilized because peak traffic happens only at specific times. With the advent of the Cloud, solution of this problem became cheaper and easier with the use of pay-per-use, flexible elastic infrastructure services provided by Cloud providers. When trying to bring these two ends together several issues, which impact the net benefit of Cloud, arise. Some of these issues are geographic distribution of user bases, capabilities of the Internet infrastructure within those geographic areas, dynamic nature of the usage patterns of the user base, and capabilities of Cloud services in terms of adaptation or dynamic reconfiguration, among others. A comprehensive study of the whole problem in the real Internet platform is extremely difficult, because it requires interaction with several computing and network elements that cannot be controlled or managed by application developers. Furthermore, network conditions cannot be predicted nor controlled, and it also impacts quality of strategy evaluation. Study of such dynamic and massively distributed environments in a controlled and reproducible manner can be achieved with the use of simulation. CloudSim ${ }^{5}$ is a Cloud simulation toolkit that allows modeling of infrastructures containing Cloud Data Centers, users, user workloads, and pricing models. CloudSim enables.

Modeling and simulation of typical Cloud infrastructures, even though it has been developed without focusing any specific Cloud provider.

CloudAnalyst Simulator: Cloud computing provides computing resources as a service over a network. As speedy function of this emerging technology in real world, it becomes more and more vital how to evaluate the performance and trust management problems that cloud computing confronts. Currently, modeling and simulation technology has become a useful and powerful tool in cloud computing research community to deal with these issues.

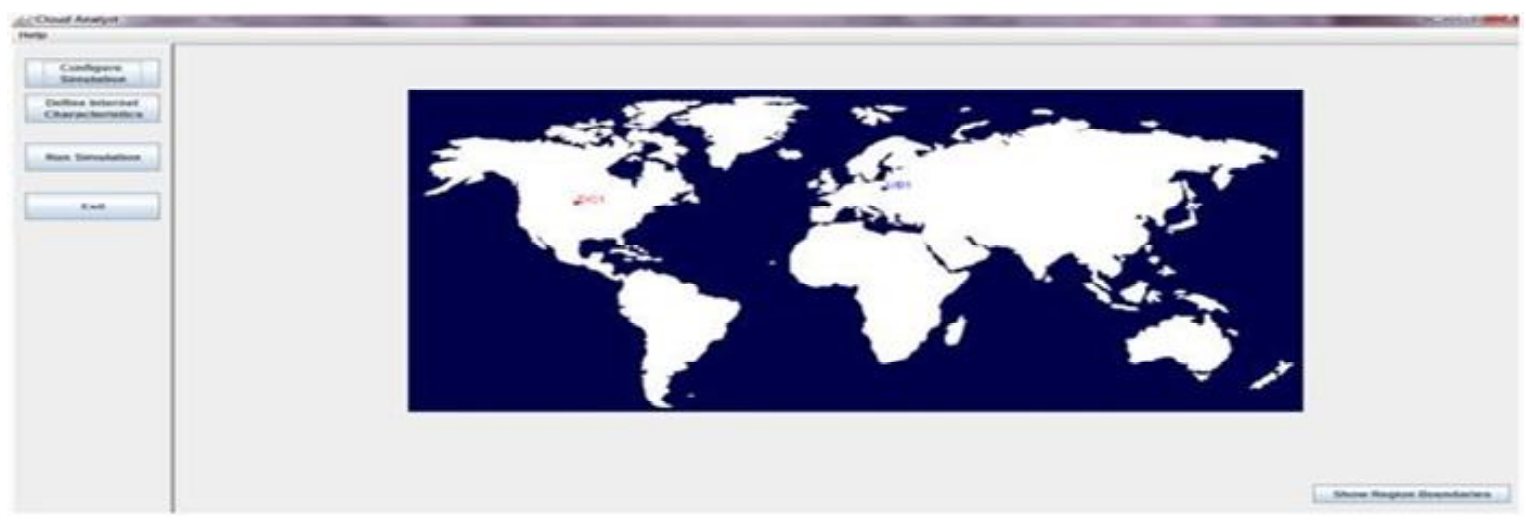

Figure 1. Cloud Analyst Main GUI Window 
Overview : As already mentioned there are several extremely good toolkits that can be used to model a simulated environment to study the behavior of a large scaled application on the Internet. But it became apparent that having an easy to use tool with a level of visualization capability is even better than just a toolkit. Such a tool separates the simulation experiment set up exercise from a programming exercise and enables a modeler to concentrate on the simulation parameters rather than the technicalities of programming.It also enables the modeler to execute simulations repeatedly with modifications to the parameters quickly and easily. A graphical output of the simulation results enables the results to be analyzed more easily and more efficiently and it may also help in quickly highlighting any problems with the performance and accuracy of the simulation logic. Therefore we decided to develop a simulation tool before starting the experiment.

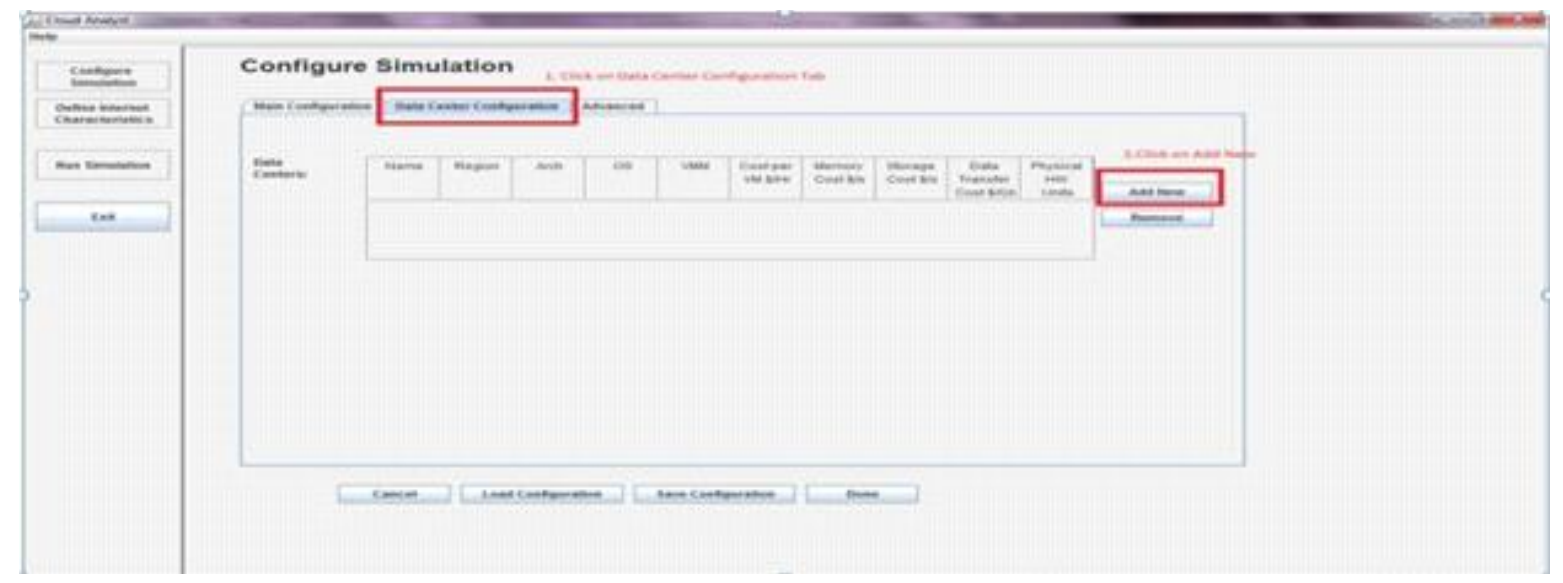

Figure 2 Physical Hardware Details of Data center

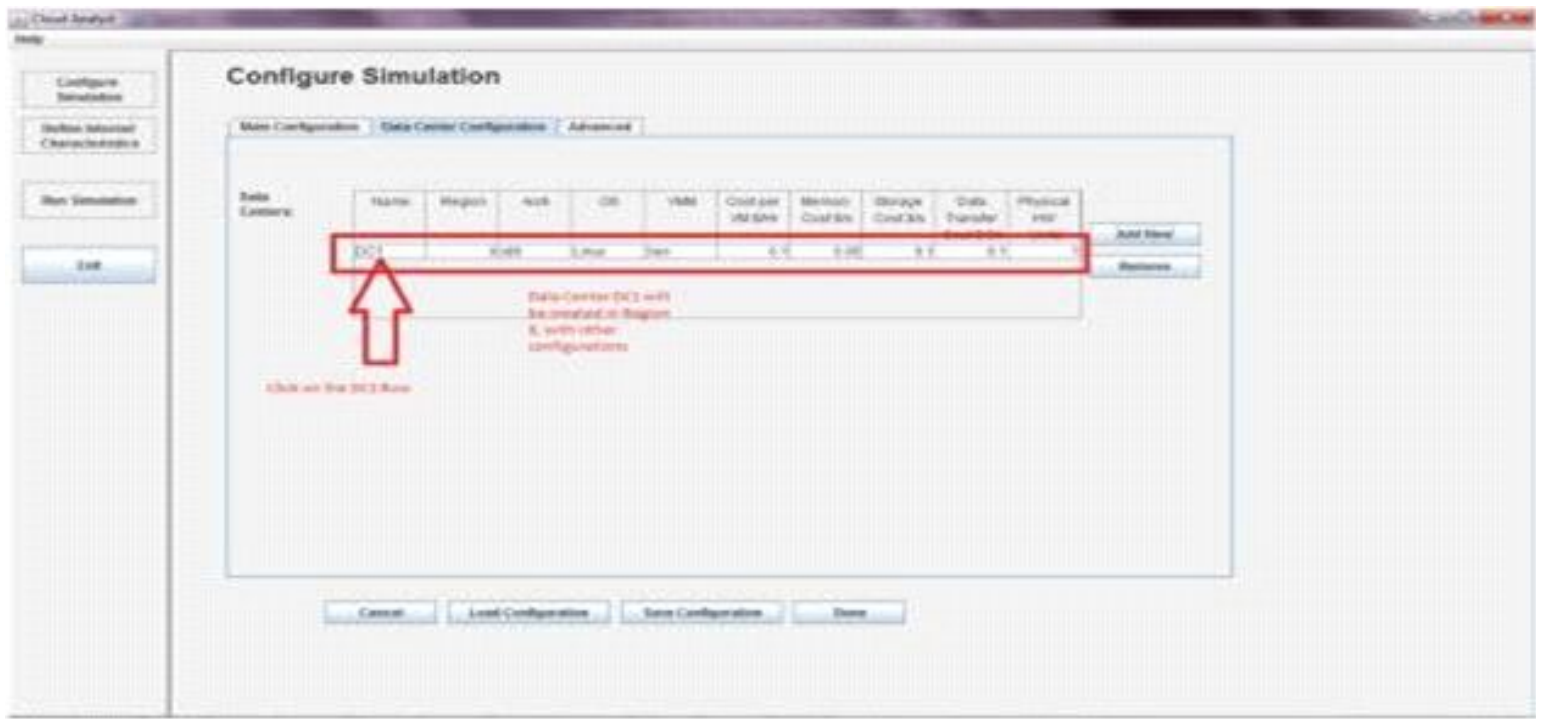

Figure 3 Cloud Analyst for Data Center Configurations 
There are several highly desirable features of a tool similar to the one described in the above section. Ease of setting up and executing a simulation experiment is the main point of having a simulation tool. The simulator needs to provide an easy to use graphical user interface which is yet comprehensive. Perhaps the most important feature is the level of configurability the tool can provide. A simulation, especially of the nature of modeling something as complex as an Internet Application depends on many paramete rs and most of the time the values for those parameters need to be assumed. Therefore it is important to be able to enter and change those parameters quickly and easily and repeat simulations.

Graphical output: A picture is said to be worth a thousand words. Graphical output in the form of tables and charts is highly desirable to summaries the potentially large amount of statistics that is collected during the simulation. S uch effective presentation helps in identifying the important patterns of the output parameters and helps in comparisons between related parameters. Repeatability of experiments is a very important requirement of a simulator. The same experiment with the same parameters should produce similar results each time the simulatio $\mathrm{n}$ is executed. Otherwise the simulation becomes just a random sequence of events rather than a controlled experiment. It is also helpful to be able to save an experiment (the set of input parameters) as a file and also be able to save the results of an experiment as a file.

Ease of extension: As already mentioned simulating something like the Internet is a complex task and it is unlikely a $100 \%$ realistic simulation framework and a set of input parameters can be achieved in a few attempts. Therefore the simu lator is expected to evolve continuously rather than a program that is written once and for all and then used continuously. Therefore the simulator architecture should support extensions with minimal effort with suitable frameworks.

Simulation Output: Following are the statistical measures produced as output of the simulation in the initial version of the simulator.

[1]. Overall average, minimum and maximum response time of all user requests simulated.

[2]. The response time broken down by user groups, located within geographical regions.

[3]. The response time further broken down by the time showing the pattern of change over the duration of a day.

\section{Usage patterns of the application:}

[1]. How many users use the application at what time from different regions of the world, and the overall effect of that usage on the data centers hosting the application. The time taken by data centers to service a user request [2]. The overall request processing time for the entire simulation.

[3]. The average, minimum and maximum request processing time by each data center.

[4]. The response time variation pattern during the day as the load changes and cost also.

\section{Technologies Used :}

[1]. Java - The simulator is developed $100 \%$ on Java platform, using Java SE 1.6.

[2]. Java Swing - The GUI component is built using Swing components.

[3]. CloudSim - CloudSim features for modeling data centers is used in CloudAnalyst. [4]. SimJava - SimJava is the underlying simulation framework of CloudSim and some

[5]. features of SimJava are used directly in Cloud Analyst.

\section{Conclusion}

With the rapid advances of $\mathrm{C}$ loud technologies, there is a new demand for tools to study and analyze the benefits of the technology and the best practices to apply the technology to large- scaled applications. Cloud Analyst is a new tool developed to address this demand. It is based on top of mature simulation frameworks such as SimJava andCloudSim. We demonstrated how C loudAnalyst can be used to model and evaluate a real world problem through a case study of a social networking application deployed on the cloud. We have illustrated how the simulator can be used to effectively identify overall usage patterns and how such usage patterns affect data centers hosting the application. Furthermore, we showed how those observations provide insights in how to optimize the deployment architecture of the application. A possibility in this direction is introduction of dynamic configurability through a global Cloud Service Broker, which increases or decreases the amount of resources available to the application in different geographic locations depending on the load at a given time.

\section{Acknowledgement}

First and foremost, we thank Lord Almighty for the grace, strength and hope to make my endeavor a success. 
It gives me immense pleasure to express my sincere thanks towards my supervisor Mrs. Suman Singh, Assistant Professor Department of Computer Science, Shekhawati Institute of Engineering \&amp; Technology, Sikar for her constant support and guidance throughout the course of this work.

\section{References}

1. Sherif Sakr, Anna Liu, Daniel M. Batista, and Mohammad Alomari, "A survey of large scale data management approaches in cloud environments", IEEE Communications Surveys \&amp, Tutorials, Vol. 13, No. 3, (2011).

2. Milani AS, Navimipour NJ. Load balancing mechanisms and techniques in the cloud environments: Systematic literature review and future trends. J N etwork Comput Appl.; 71, 86-98 (2016).

3. Armbrust, M., Above the clouds: A berkeley view of cloud computing. Tech. Rep. UCB/EECS-2009-28, EECS Department, U.C. Berkeley, Feb (2009).

4. Greenberg A, Jain N, et al., VL2: a scalable and flexible data center network. In: Proc SIGCOMM (2009).

5. Duncan, Dexter \&amp; C hu, Xingchen \&amp;
Vecchiola, C hristian \&amp; Buyya, Rajkumar. (2018), The Structure of the New IT Frontier: Cloud Computing- Part I.

6. Buyya, R., Yeo, C. S., \&amp; Venugopal, S. (2008, September). Market-oriented cloud computing: Vision, hype, and reality for delivering it services as computing utilities. In High Performance Computing and Communications, 2008. HPCC\&\#39;08. 10th IEEE International Conference on (pp. 5-13).

7. Buyya, R., Yeo, C. S., Venugopal, S., Broberg, J., \&amp; Brandic, I., Cloud computing and emerging IT platforms: Vision, hype, and reality for delivering computing as the 5th utility. Future Generation computer systems, 25(6), 599-616 (2009).

8. Habib, S. M., Ries, S., \&amp; Muhlhauser, M. (2011, November). Towards a trust management system for cloud computing. In Trust, Security and Privacy in Computing and Communications (TrustCom), 2011 IEEE 10th International Conference on (pp. 933-939). IEEE.

9. Jøsang, A., Ismail, R., \&amp; Boyd, C., A survey of trust and reputation systems for online service provision. Decision support systems, 43(2), 618644 (2007). 